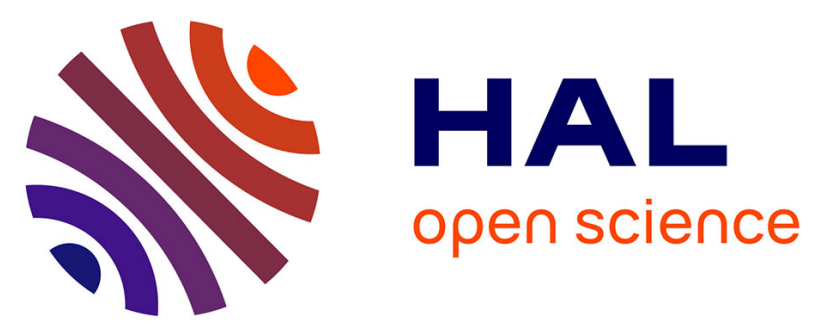

\title{
Investigation of the spontaneous nanoemulsification process with medium- and long-chain triglycerides
}

Yasmine Jamoussi, Taghrid Zaiter, Catherine C. Desrumaux, Niyazi Acar, Yann Pellequer, Arnaud Béduneau

\section{- To cite this version:}

Yasmine Jamoussi, Taghrid Zaiter, Catherine C. Desrumaux, Niyazi Acar, Yann Pellequer, et al.. Investigation of the spontaneous nanoemulsification process with medium- and long-chain triglycerides. Colloids and Surfaces B: Biointerfaces, 2021, 197, pp.111432. 10.1016/j.colsurfb.2020.111432 . hal03034581

\section{HAL Id: hal-03034581 \\ https://hal.inrae.fr/hal-03034581}

Submitted on 1 Dec 2020

HAL is a multi-disciplinary open access archive for the deposit and dissemination of scientific research documents, whether they are published or not. The documents may come from teaching and research institutions in France or abroad, or from public or private research centers.
L'archive ouverte pluridisciplinaire HAL, est destinée au dépôt et à la diffusion de documents scientifiques de niveau recherche, publiés ou non, émanant des établissements d'enseignement et de recherche français ou étrangers, des laboratoires publics ou privés.

\section{()ㅜ) $\Theta$}

Distributed under a Creative Commons Attribution - NoDerivatives| 4.0 International 


\title{
Investigation of the spontaneous nanoemulsification process with medium- and long-chain triglycerides
}

\author{
Yasmine Jamoussi ${ }^{a}$, Taghrid Zaiter ${ }^{a}$, Catherine Desrumaux ${ }^{b}$, Niyazi Acar ${ }^{c}$, Yann Pellequer ${ }^{a}$, \\ Arnaud Béduneau ${ }^{\text {a, * }}$ \\ ${ }^{a}$ PEPITE EA4267, Labex LipSTIC, Univ. Bourgogne Franche-Comté, F-25000 Besançon, France \\ ${ }^{\mathrm{b}}$ Molecular Mechanisms in Neurodegenerative Dementia Laboratory (MMDN), INSERM, U1198, Environmental Impact in Alzheimer's Disease and Related Disorders \\ (EiAlz) Team, 34095 Montpellier, France; University of Montpellier, 34095 Montpellier, France; EPHE, 75014 Paris, France \\ ${ }^{\mathrm{c}}$ Centre des Sciences du Goût et de l'Alimentation, AgroSup Dijon, CNRS, INRAE, Université Bourgogne Franche-Comté, Eye and Nutrition Research Group, Dijon, F- \\ 21000, France
}

\section{A R T I C L E I N F O}

\section{Keywords:}

Nanoemulsions

Triglycerides

Vegetable oils

Low-temperature process

Spontaneous emulsification

Ostwald ripening

\begin{abstract}
A B S T R A C T
Oil-in-water nanoemulsions are used in numerous biomedical applications as delivery systems. The droplet size in the nanometer range and their composition were extensively developed for carrying and enhancing the absorption of lipophilic drugs and lipids of interest. In the present study, critical parameters involved in the spontaneous nanoemulsification process such as the temperature, the oil type, the surfactant-to-oil and water-tooil ratios were investigated. The aim was to design a solvent-free procedure for the spontaneous nanoemulsification at a low temperature of a large variety of triglycerides including vegetable oils. Nanoemulsification of medium-chain triglyceride (MCT) was not dependent on the temperature while nanodroplets of long-chain triglycerides (LCT) were only obtained by reaching the cloud point of ethoxylated surfactant Kolliphor ${ }^{\circledR}$ HS15. The molar volume of triglycerides was considered as a predictive parameter governing both, the spontaneous nanoemulsification at low temperature and the Ostwald ripening rate. The physical mixture of MCT and LCT was a promising strategy to prepare stable and fine nanoemulsions at $37^{\circ} \mathrm{C}$. They were characterized by a hydrodynamic diameter comprised between 20 and $30 \mathrm{~nm}$ and a narrow size distribution. These findings pave the way to new applications for the parenteral nutrition and the delivery of thermosensitive drugs and lipophilic molecules such as antioxidants.
\end{abstract}

\section{Introduction}

Water-in-oil nanoemulsions are colloidal lipid systems characterized with sizes below $200 \mathrm{~nm}$ [1]. Preparation processes are classified according to the energy required to reduce the size of oil droplets. High-energy methods including homogenizers and microfluidic devices generate high shear rates to expand the surface of oil droplets in water. Low-energy processes include phase inversion temperature methods (PIT) and spontaneous emulsification termed in some applications self-emulsifying methods. Then PIT method was first investigated by Shinoda et al. [2]. It consists in heating the preparation at temperatures above the PIT to convert an oil/water emulsion to a water/oil emulsion passing through a bicontinuous phase. A rapid dilution is then performed, leading to the formation of nanodroplets. The mechanism is based on the temperature-dependent solubility of ethoxylated surfactants in oil and water. The PIT corresponds to an equal solubility of surfactant for water and oil. The spontaneous emulsification or self-emulsifying methods is a low-energy method, allowing the rapid formation of oil droplets in the nanometer range. The organic phase is usually composed of oil, surfactant and water-miscible solvent termed co-solvent such as acetone and ethanol [3]. Various mechanisms were suggested to explain the nanoemulsion formation after mixing of two immiscible phases ; (a) organic phase diffusion in water [4]; (b) surfactant migration from the organic phase to the oil/water interface [5]; (c) water transfer in the organic phase leading to the surfactant solubilization and the fragmentation of the oil phase [6]. Besides the ease of preparation process, another advantage is the possibility to produce a nanoemulsion directly in the body after contact of the lipid phase with biologic fluids [7].

Nanoemulsions were widely used in biomedical applications. They

\footnotetext{
* Corresponding author.

E-mail address: arnaud.beduneau@univ-fcomte.fr (A. Béduneau).
} 
can deliver class II drugs according to the biopharmaceutical classification characterized by a poor water solubility. Class IV drugs were also loaded in nanoemulsions due to their ability to cross epithelia such as the intestinal barrier [8,9]. In addition to the size in the nanometer range, drug absorption is promoted by the presence of surfactants which can modulate the efflux transporters [10]. Antioxidant agents including curcumin, $\alpha$-tocopherol, and polyunsaturated lipids were loaded in nanoemulsions [11]. Nanoemulsions composed of lipid mixtures were also developed for the parenteral nutrition. Within them, Lipidem ${ }^{\circledR}$ is composed of medium chain triglycerides (MCT), omega 3 polyunsaturated fatty acids and soybean triglycerides [12].

Despite the extensive studies dedicated to nanoemulsions, their development faced numerous problems. Firstly, high temperatures required for the PIT method [13] or resulting from the mechanical energy dissipation in high-energy processes can alter the chemical stability of drugs and lipophilic molecules such as antioxidants. Secondly, large quantities of co-solvents such as acetone and ethanol are often necessary to reduce the size of oil droplets in spontaneous emulsification methods [3]. They can be responsible for adverse effects, especially in in situ emulsification strategies such as oral lipid-based delivery systems where they cannot be removed [14]. In addition, co-solvent diffusion in water can trigger drug precipitation [7]. Third, the choice of excipients, and especially oils and surfactants, is often limited in low-energy processes [4], reducing the applications of nanoemulsions to lipid and drug delivery systems $[15,16]$. The last point concerns the long-term stability of nanoemulsions that represents a real challenge. The Ostwald ripening (OR) is the main mechanism responsible for the destabilization of nanoemulsions due to Laplace pressure differences between small and large droplets [1]. It consists in the migration of individual oil molecules from small to larger droplets by diffusion through the continuous aqueous phase. OR rate depends on the size distribution of droplets and the nature of the oil and can be affected by the presence of micelles in the continuous phase [17-19].

In the present study, the influence of the process temperature and of material attributes on the size and the stability of oil/water nanoemulsions was investigated. The aim was to design a low temperature nanoemulsification process without organic water-miscible solvent, allowing the delivery of a wide variety of triglycerides including MCT and long-chain triglycerides (LCT) as well as thermosensitive drugs and lipophilic molecules.

\section{Materials and methods}

\subsection{Materials}

Glyceryl trioleate (96\%) was provided by Santa Cruz Biotechnology (Heidelberg, Germany). Glyceryl trioctanoate (99\%), corn oil, olive oil and Kolliphor ${ }^{\circledR}$ HS15 (macrogol 15 hydroxystearate) were supplied by Sigma-Aldrich (Saint Quentin Fallavier, France). Labrafac WL 1349 was a gift of Gattefossé SAS (Saint Priest, France).

\subsection{Nanoemulsion preparation}

Lipid phase consisted of a mixture of Kolliphor® HS15 (Macrogol 15 hydroxystearate) with different oils including Labrafac WL 1349, vegetable oils, glyceryl trioctanoate or glyceryl trioleate (Table 1). Oils were composed mainly of triglycerides with different fatty chain lengths (Table 1). The surfactant-to-oil ratio (SOR) ranged from 1 to 6. Lipid phase and deionized water were separately heated under magnetic stirring in a water bath at equal temperatures comprised between $37^{\circ} \mathrm{C}$ and $90{ }^{\circ} \mathrm{C}$ during $10 \mathrm{~min}$. Water was then rapidly added in the lipid phase using a syringe at a water-to-oil ratio (WOR) ranging from 5 to 100. The two immiscible phases were magnetically stirred in the water bath at the target temperature for $5 \mathrm{~min}$. The preparation was then removed from the water bath and stirred at room temperature for 15 min. Approximately $7.5 \mathrm{~g}$ of emulsion were prepared for each batch.
Table 1

Description of the oils used for the preparation of nanoemulsions.

\begin{tabular}{lllll}
\hline Oil & Composition & Density & Mw & $\begin{array}{l}\text { Viscosity at } \\
25^{\circ} \mathrm{C}(\mathrm{mPa} . \mathrm{s})\end{array}$ \\
\hline Corn oil (CO) & $\begin{array}{l}\text { C18:1 (29\%); } \\
\text { C18:2 (56\%) }\end{array}$ & 0.9 & - & $54.0 \pm 0.5$ \\
$\begin{array}{l}\text { C8:0 (99\%) } \\
\begin{array}{l}\text { Glyceryl } \\
\text { trioctanoate (GT) }\end{array}\end{array}$ & 0.956 & 470.7 & $21.6 \pm 0.0$ \\
$\begin{array}{c}\text { Glyceryl trioleate } \\
\text { (GTOL) }\end{array}$ & C18:1 (96\%) & 0.915 & 885.4 & $65.6 \pm 1.1$ \\
$\begin{array}{c}\text { Labrafac WL 1349 } \\
\quad \text { Labrafac) }\end{array}$ & $\begin{array}{l}\text { C8:0 (56\%); C10:0 } \\
(43 \%)\end{array}$ & 0.946 & 506.2 & $25.4 \pm 0.2$ \\
$\begin{array}{l}\text { Olive oil (OO) } \\
\text { C18:1 (61 \%); }\end{array}$ & 0.912 & - & $67.5 \pm 0.1$ \\
\hline
\end{tabular}

\subsection{Particle size measurements}

The hydrodynamic and the polydispersity index (PDI) of emulsions were determined by dynamic light scattering using a Zetasizer Nano ZS 90, (Malvern Instruments, Orsay, France). The apparatus was equipped of a 4-mW HeNe laser at a wavelength of $632.8 \mathrm{~nm}$. Light scattering was detected at an angle of $90^{\circ}$. The temperature was fixed at $25^{\circ} \mathrm{C}$. All measurements were performed in triplicate after a 1:10 dilution in deionized water.

\subsection{Viscosity measurements}

Viscosity of oils was measured using a Kinexus Pro rheometer in controlled shear mode (Malvern Instruments, Orsay, France). Measuring systems were composed of a cone with a $2^{\circ}$ angle and a $50 \mathrm{~mm}$ diameter plate. Temperature was controlled using a Peltier system. A solvent trap device was used during the heating process. The apparent shear viscosity was measured at temperatures ranging from $25{ }^{\circ} \mathrm{C}$ to $90{ }^{\circ} \mathrm{C}$ using a steady state flow process at a shear rate of $100 \mathrm{~s}^{-1}$. At least two independent measurements were performed for each type of oil.

\section{Results and discussion}

\subsection{Influence of temperature}

The nanoemulsification process was performed at $37{ }^{\circ} \mathrm{C}$ using MCT such as glyceryl trioctanoate, Labrafac WL 1349 and LCT including corn oil, olive oil and glyceryl trioleate. They were selected for their low melting point, allowing the synthesis of nanodroplets with a liquid lipid core. The surfactant-to-oil ratio (SOR) and the water-to-oil ratio (WOR) were first fixed at 3.5 and 22, respectively. In Fig. 1, only small nanodroplets were obtained with glyceryl trioctanoate and Labrafac WL 1349 with sizes below $25 \mathrm{~nm}$ and a polydispersity index (PDI) close to 0.1, demonstrating a monomodal distribution. This was consistent with the study of Lefevre et al. which demonstrated the spontaneous nanoemulsification of Labrafac at $30{ }^{\circ} \mathrm{C}$ and $50{ }^{\circ} \mathrm{C}$ [5]. For glyceryl trioleate, corn and olive oils, sizes and PDI were much larger than $100 \mathrm{~nm}$ and 0.1, respectively. These data demonstrate that the nanoemulsification process is clearly dependent on the type of oil, especially the hydrocarbon chain length. The glyceryl trioctanoate and the Labrafac are composed of fatty acids with a carbon chain length $\leq 10$ whereas LCT contain C18 fatty acids.

The emulsification mechanism could be affected by physicochemical properties of triglycerides such as the viscosity and the tension at the oil/ water interface. However, literature showed interfacial tension of vegetable oils and MCT in water were within the same range, comprised between 23 and $25 \mathrm{mN} \cdot \mathrm{m}^{-1}$ [20]. Thus, the influence in this parameter was not investigated in the present study. Wooster et al. demonstrated that the oil viscosity plays a major role in the size of droplets. Heighty $\mathrm{nm}$ and $120 \mathrm{~nm}$ nanoemulsions were obtained with hexadecane and LCT, respectively [21]. Viscosities of triglycerides and lipid mixtures 


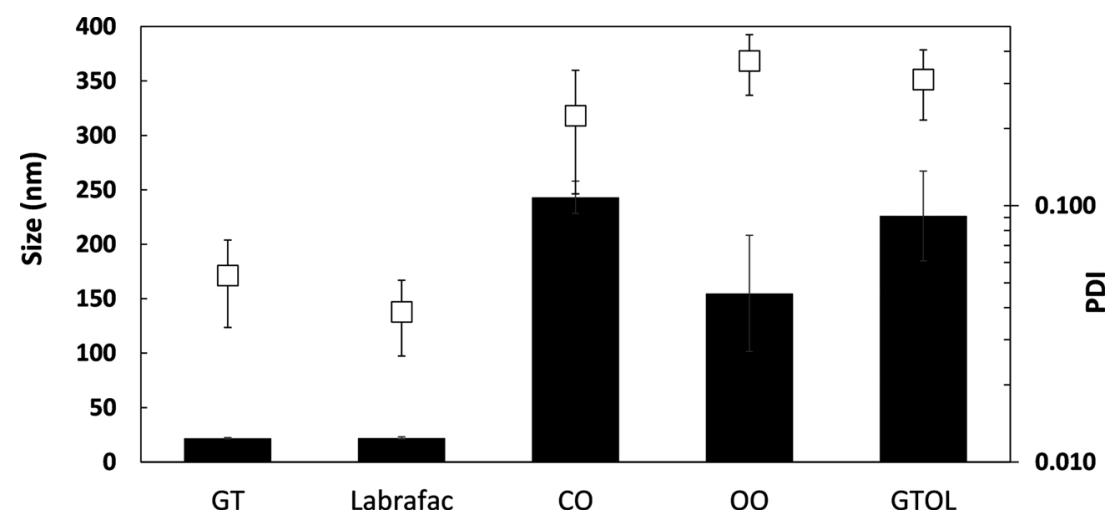

Fig. 1. Influence of the oil type on the size (black bars) and the PDI (open squares) of nanoemulsions prepared at $37{ }^{\circ} \mathrm{C}$ with a SOR and a WOR of 3.5 and 22 , respectively. Formulations were prepared with glyceryl trioctanoate (GT), Labrafac WL 1349 (Labrafac), corn oil (CO), olive oil (OO) and glyceryl trioleate (GTOL). Results are expressed as mean values \pm SD. Measurements were performed in triplicate from at least three independent experiments.

were measured at a shear rate of $100 \mathrm{~s}^{-1}$. In Fig. 2, viscosity values of glyceryl trioctanoate and Labrafac at $37^{\circ} \mathrm{C}$ were respectively of 13.2 and $15.8 \mathrm{mPa}$.s whereas the values were of 34.8 and $41.7 \mathrm{mPa}$.s for corn and olive oils, respectively. Heating process decreased drastically the viscosity of oils. At $70{ }^{\circ} \mathrm{C}$, corn oil and olive oils reached values of 13 and $14.6 \mathrm{mPa} . \mathrm{s}$, in the same range as glyceryl trioctanoate and Labrafac at 37 ${ }^{\circ} \mathrm{C}$.

To investigate the role of viscosity, nanoemulsification of LCT was performed at temperatures higher than $37^{\circ} \mathrm{C}$ such as $50^{\circ} \mathrm{C}, 70^{\circ} \mathrm{C}$ and 90 ${ }^{\circ} \mathrm{C}$ (Fig. 3). Nanodroplets with diameters of approximately $25 \mathrm{~nm}$ were only obtained at $90^{\circ} \mathrm{C}$ for all the triglycerides. Between $37^{\circ} \mathrm{C}$ and $70^{\circ} \mathrm{C}$, no clear evidence of a temperature-dependent effect was observed on the size of nanoemulsions. The polydispersity indexes were above 0.1 , suggesting a heterogeneous size distribution. Despite the viscosity drop during the heating and values close to MCT at $70^{\circ} \mathrm{C}$, the heating did not significantly reduce the size of nanodroplets except at $90{ }^{\circ} \mathrm{C}$. This result suggests the lack of relationship between the viscosity of oils and the size of nanoemulsions for the spontaneous nanoemulsification.

Besides the viscosity of triglycerides, temperature variations affect also the partition coefficient of ethoxylated surfactants in oil and water [22]. It was reported that temperature increase drastically reduces the aqueous solubility of nonionic surfactant [23]. The cloud point of macrogol 15 hydroxystearate in pure water was observed at a temperature of $85{ }^{\circ} \mathrm{C}$ [24]. By considering the correlation between the PIT and the cloud point demonstrated by Shinoda et al. [25], it could be reasonably assumed that the heating at $90^{\circ} \mathrm{C}$ with LCT allowed reaching the PIT corresponding to an equal solubility of surfactant in oil and water and the formation of a bicontinuous system [26,27]. However, conductimetry measurements performed during the heating did not demonstrate a phase inversion likely due to the high water-to-oil ratio of 22:1.

In the case of MCT, the spontaneous emulsification did not require reaching the PIT. As discussed previously, the interfacial tension and the viscosity does not justify this difference. In presence of organic watermiscible solvent, spontaneous emulsification is usually reported as a diffusion driven process [28]. Due to the lack of co-solvent in our preparations, emulsification could be explained by the rapid diffusion of MCT in the aqueous phase. MCT are well known to be more soluble than LCT due shorter fatty acid chains [29]. Their solubility could be also improved by the presence of Kolliphor ${ }^{\circledR}$ HS15. Taylor et al. showed that the diffusion coefficient D of oil molecules including alkanes was calculated according to the following equation [30]:

$D=7.4 \times 10^{-12} \times \frac{\left(\Phi_{s} m_{1}\right)^{0.5} T}{\eta_{1} V_{2}^{0.6}}$

$\mathrm{m}_{1}$ and $\eta_{1}$ are defined as the molar mass $(\mathrm{g} / \mathrm{mol})$ and the viscosity ( $\mathrm{mPa}$. s) of the solvent. $V_{2}$ is the molar volume of the diffusate $\left(\mathrm{cm}^{3} / \mathrm{mol}\right)$ and is calculated by dividing the molar mass by the mass density. $\Phi$ s is the association factor of the solvent allowing molecule interactions such as

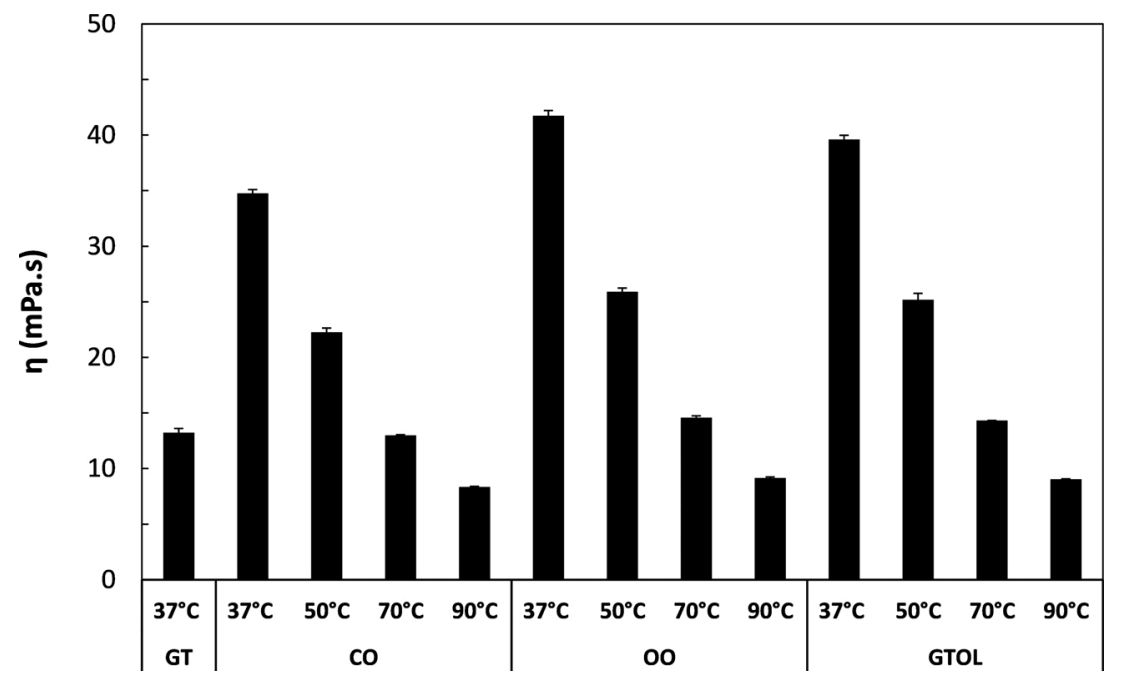

Fig. 2. Influence of the temperature on the viscosity of different oils including glyceryl trioctanoate (GT), corn oil (CO), olive oil (OO) and Glyceryl trioleate (GTOL) at a shear rate of $100 \mathrm{~s}^{-1}$. Results are expressed as mean values $\pm \mathrm{SD}$. Measurements were performed in triplicate from at least two independent experiments. 


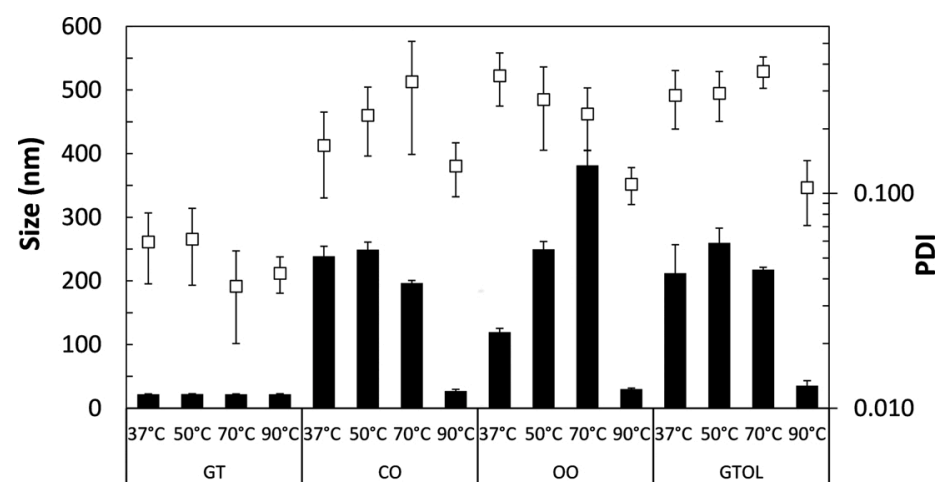

Fig. 3. Influence of the process temperature on the size (black bars) and the PDI (open squares) of nanoemulsions prepared with glyceryl trioctanoate (GT), corn oil (CO), olive oil (OO) and glyceryl trioleate (GTOL). Results are expressed as mean values \pm SD. Measurements were performed in triplicate from at least two independent experiments.

hydrogen bonds. According to Eq. 1, the molar volume (Vm) would be the main intrinsic property of the oil phase which controls the diffusion coefficient in the water bulk phase. Considering the narrow density range of triglycerides $(0.9-0.95)$, Vm could be also associated to the water solubility of MCT and LCT which depends of the length of fatty acids and consequently of the molecular weight [29]. This was in agreement with Wooster et al. who stated that the water solubility of an oil is inversely proportional to $\mathrm{Vm}$ [21]. By predicting both the aqueous solubility and the diffusion coefficient in water, the molar volume of triglycerides could be a critical parameter for the spontaneous nanoemulsification. This would explain the spontaneous nanoemulsification at $37{ }^{\circ} \mathrm{C}$ of glyceryl trioctanoate characterized a Vm of $492 \mathrm{~cm}^{3} / \mathrm{mol}$ unlike glyceryl trioleate exhibiting a value of $968 \mathrm{~cm}^{3} / \mathrm{mol}$. Vm was not calculated for vegetable oils due to their complex composition. However, considering the high proportion of triglycerides in vegetable oils and by taking into account the preponderant C18 fatty acids, the value should be in the same range as that of glyceryl trioleate [31]. Thus, the higher water solubility and diffusion coefficient of MCT suggested by their low Vm compared to LCT could explain their spontaneous and rapid dispersion into nanodroplets at $37^{\circ} \mathrm{C}$.

\subsection{Influence of the lipid composition}

The high temperature required for the spontaneous nanoemulsification of long chain triglycerides could affect the chemical stability of lipids, and especially hat of unsaturated fatty acids. To facilitate the oil diffusion during the spontaneous emulsification at lower temperatures, water-miscible organic solvents termed as co-solvents such as acetone and ethanol are usually added in the lipid phase [3]. Our previous results demonstrated that MCT can diffuse easily in the external water phase and then could play the role of a co-solvent. To investigate their interest in the nanoemulsification process, glyceryl trioctanoate was added with LCT including glyceryl trioleate, olive and corn oils and at a ratio 1:1 (Fig. 4). SOR and WOR were first at 3.5 and approximately 22, respectively. Nanoemulsification process was performed at $37^{\circ} \mathrm{C}$. Interestingly, oil nanodroplets with a size below $25 \mathrm{~nm}$ were obtained for the 3 lipids mixtures. In addition, the polydispersity index was inferior to 0.1, suggesting the formation of a single population of nanodroplets.

\subsection{Influence of SOR and WOR}

To investigate the effect of the surfactant concentration on the size and the polydispersity of nanodroplets, nanoemulsions with different SOR comprised between 6 and 1 were prepared. Process temperature was $37^{\circ} \mathrm{C}$ and WOR was fixed at 22 . Nanoemulsions were prepared with different oil types including glyceryl trioctanoate, Labrafac, corn oils and a GT/CO mixture at a ratio 1:1. In Fig. 5A and B, nanoemulsions

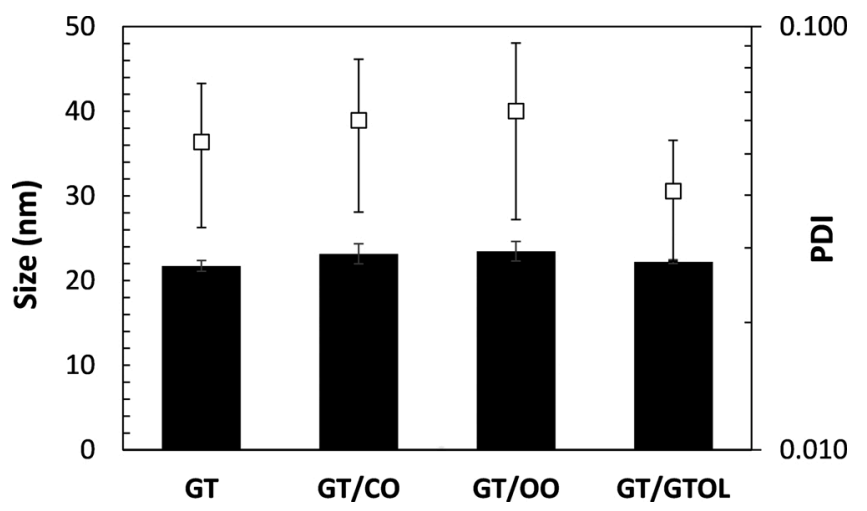

Fig. 4. Influence of the LCT/LGT mixture on the size (black bars) and PDI (open squares) of nanoemulsions prepared at $37{ }^{\circ} \mathrm{C}$ with a SOR of 3.5 and WOR of 22. Glyceryl trioctanoate was mixed at ratio 1:1 with corn oil (GT/CO), olive oil (GT/OO) and glyceryl trioleate (GT/GTOL). Results are expressed as mean values \pm SD. Results were compared with pure glyceryl trioctanoate (GT). Measurements were performed in triplicate from at least three independent experiments.

prepared at a SOR 6 were characterized by a size close to $20 \mathrm{~nm}$ for every oil type and a PDI close to 0.1. Interestingly, no heating was then necessary for the preparation of oil corn nanoemulsions at that SOR value. This could be due to the oil solubilization in Kolliphor ${ }^{\circledR}$ HS15 micelles. At a SOR 5, corn oil droplets reached a size of $173 \mathrm{~nm}$ and a PDI of 0.3. Regarding MCT and the mixture GT/CO, no significant size increase was observed for SOR comprised between 6 and 3 . Hydrodynamic diameters was still below $30 \mathrm{~nm}$ and PDI close to 0.1 . The size of the emulsion prepared with the lipid mixture increased drastically from a SOR of 2, reaching $70 \mathrm{~nm}$. The PDI of 0.370 suggested a multimodal distribution. The same behavior was observed with MCT. Both size and PDI increases were observed between SOR 2 and 1 . At SOR 1 , sizes were approximately of $90 \mathrm{~nm}$ and the PDI above 0.3 . This was consistent with the work of Lefebvre et al. showing a significant influence of SOR on the size and the polydispersity of nanoemulsions prepared with Labrafac [5]. Influence of WOR on the size of nanoemulsions prepared at $37{ }^{\circ} \mathrm{C}$ and composed of glyceryl trioctanoate alone or with corn oil were also investigated (Fig. 6). WOR comprised between 5 and 100 did not affect significantly the size of droplets with values close to $22 \mathrm{~nm}$ for MCT and between $24 \mathrm{~nm}$ and $22 \mathrm{~nm}$ for the triglyceride mixture. PDI was inferior to 0.1 except for the nanoemulsions prepared with the GT/CO mixture at a WOR 5. Nevertheless, the value was of 0.178 , suggesting a narrow size distribution. In addition, the way to add together the two immiscible phases did not change the nanoemulsion properties. A size close to 20 $\mathrm{nm}$ and a monomodal size distribution were obtained after addition at 

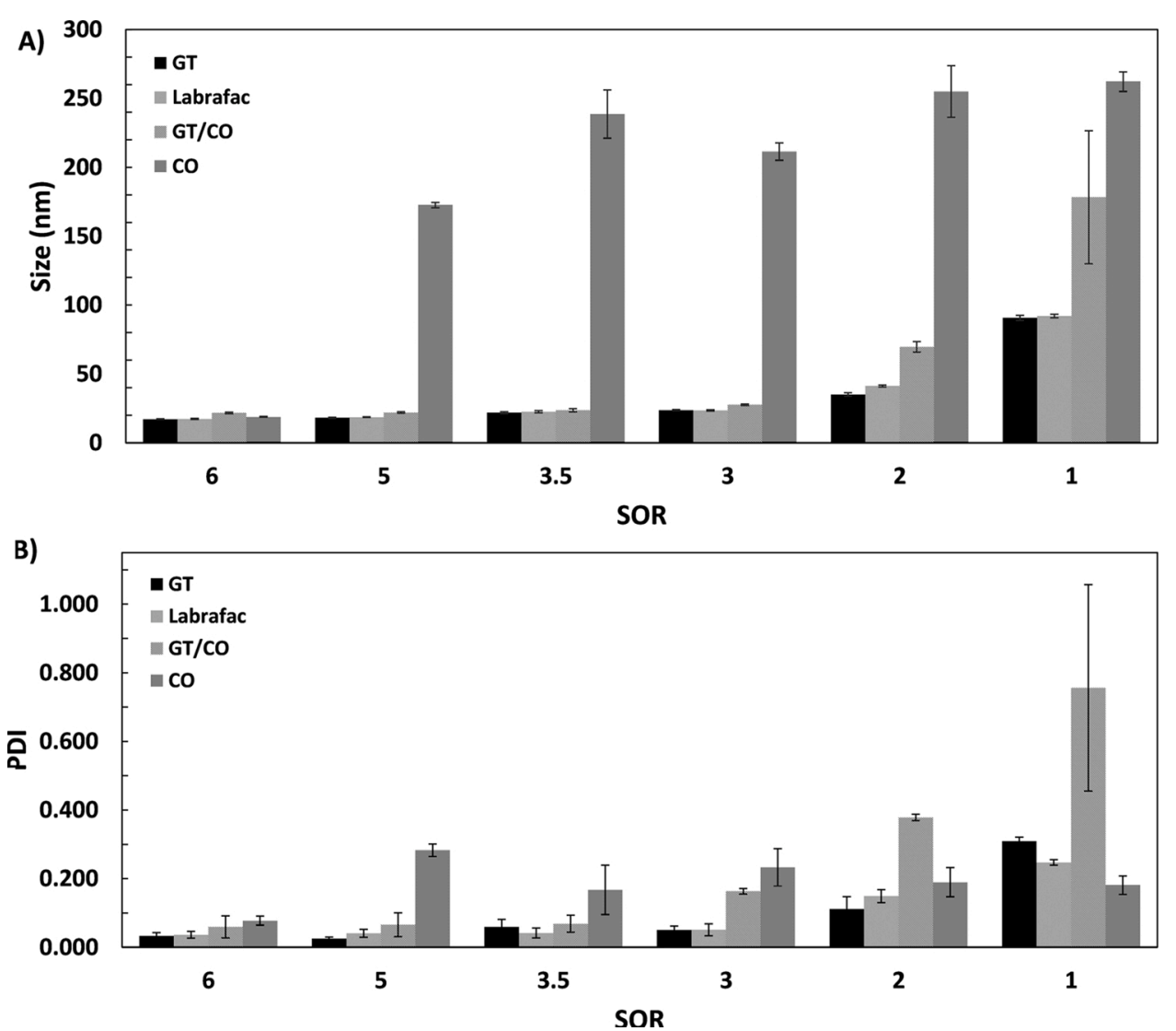

Fig. 5. Influence of surfactant-to-oil ratio on the nanoemulsification of glyceryl trioctanoate (GT), Labrafac WL 1349 (Labrafac) and 1:1 lipid mixtures composed of GT with corn oil (GT/CO) or olive oil (GT/OO). Preparations were performed at $37{ }^{\circ} \mathrm{C}$ and the water-to-oil ratio was fixed at 22. A: hydrodynamic diameters of oil droplets as a function of the SOR. B: PDI of nanoemulsions as a function of the SOR. Results are expressed as mean values \pm SD. Measurements were performed in triplicate from at least two independent experiments.

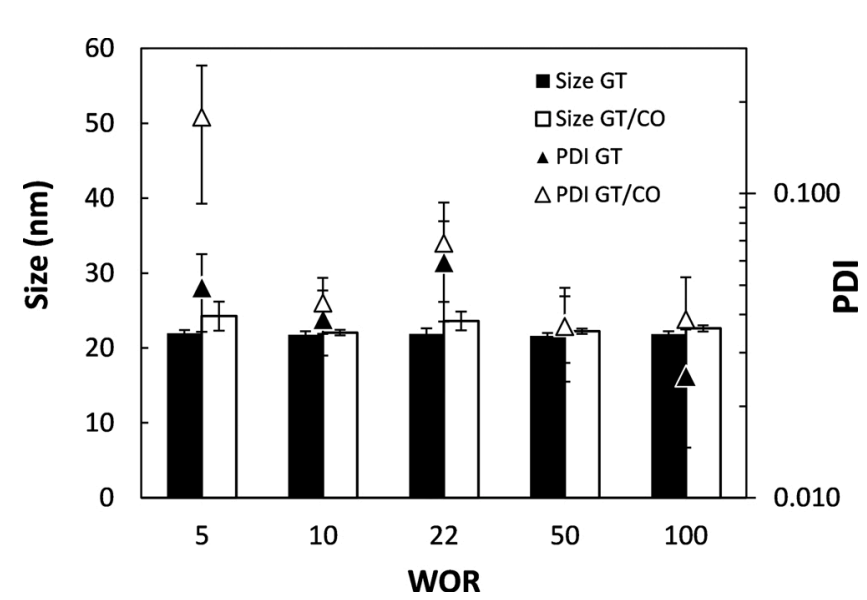

Fig. 6. Influence of water-to-oil ratio (WOR) on the size and the PDI of nanoemulsions prepared at $37{ }^{\circ} \mathrm{C}$. SOR was fixed at 3.5. Lipid phase was composed of glyceryl trioctanoate (GT) or of a 1:1 mixture of GT and corn oil (GT/CO). Results are expressed as mean values \pm SD. Measurements were performed in triplicate from at least two independent experiments.

$37{ }^{\circ} \mathrm{C}$ of lipid phases composed of GT and a GT/CO mixture in water. This data appears as very interesting for the preparation of nanoemulsion after dilution in the biologic fluids.

\subsection{Stability}

The stability of nanoemulsions stored at $4{ }^{\circ} \mathrm{C}$ was evaluated over time (Fig. 7A-C). Except for pure glyceryl trioctanoate nanoemulsions, lipid mixtures, Labrafac and LCT were stable during 90 days. No significant size increase was observed and PDI stayed close to 0.1, suggesting a monomodal size distribution. Regarding GT nanoemulsions, no significant size increase was observed within the first days followed by a rapid growth of oil droplets. After the lag-phase, the cube of droplet radius increased linearly with time, suggesting a destabilization mechanism governed by the Ostwald ripening. This was confirmed by the PDI values, which increased at the beginning of the destabilization kinetic to finally drop to values inferior to 0.2 , corresponding to the end of the droplet growth. Although it was reported that surfactant can increase the Ostwald ripening rate due to the oil solubilization in micelles [18], a retardation of the Ostwald ripening was also observed by Weiss et al. for high concentrations of non-ionic surfactants (above $10 \%$ ) [19]. This could be due to the high ability of micelles to solubilize large quantities of oil. Besides, according to the theory of Lifshitz, Slyozov and Wagner termed the LSW theory, the OR rate increases with the diffusion coefficient and the bulk solubility of the oil, and consequently could be also correlated to $\mathrm{Vm}[17,32]$. It would explain the rapid destabilization of nanoemulsions containing the GT that is characterized by the lowest Vm among the studied triglycerides including MCT and LCT. This hypothesis was supported by a study showing that glyceryl trioctanoate and mygliol, a MCT composed of a mixture of C8 and C10 fatty acids chains were characterized by an OR rate of 0.345 and $0.0483 \mathrm{~nm}^{3} / \mathrm{s}$, respectively for $\mathrm{Vm}$ of 508 and 614 $\mathrm{cm}^{3} / \mathrm{mol}$ [21]. In the same article, no Ostwald ripening was observed over 3 months with peanut oils composed of long fatty acid chains (> C18). Unlike GT, stability of Labrafac nanoemulsions could be explained by the presence of $43 \%$ of $\mathrm{C} 10$ fatty acids with $56 \%$ of C 8 . Thus, the molar volume of Labrafac equal to $535 \mathrm{~cm}^{3} / \mathrm{mol}$ could be considered as a target value allowing both the spontaneous emulsification and the stability. Interestingly, the mixture of LCT with GT at a ratio $1 / 1$ were still stable after 90 days. Higushi et al. demonstrated that the addition of insoluble oils enhances nanoemulsion stability by reducing the Ostwald ripening rate [33]. From a molar fraction of insoluble oil equal to 0.4 , nanoemulsions composed of glyceryl trioctanoate and peanut oil were 
A)

B)
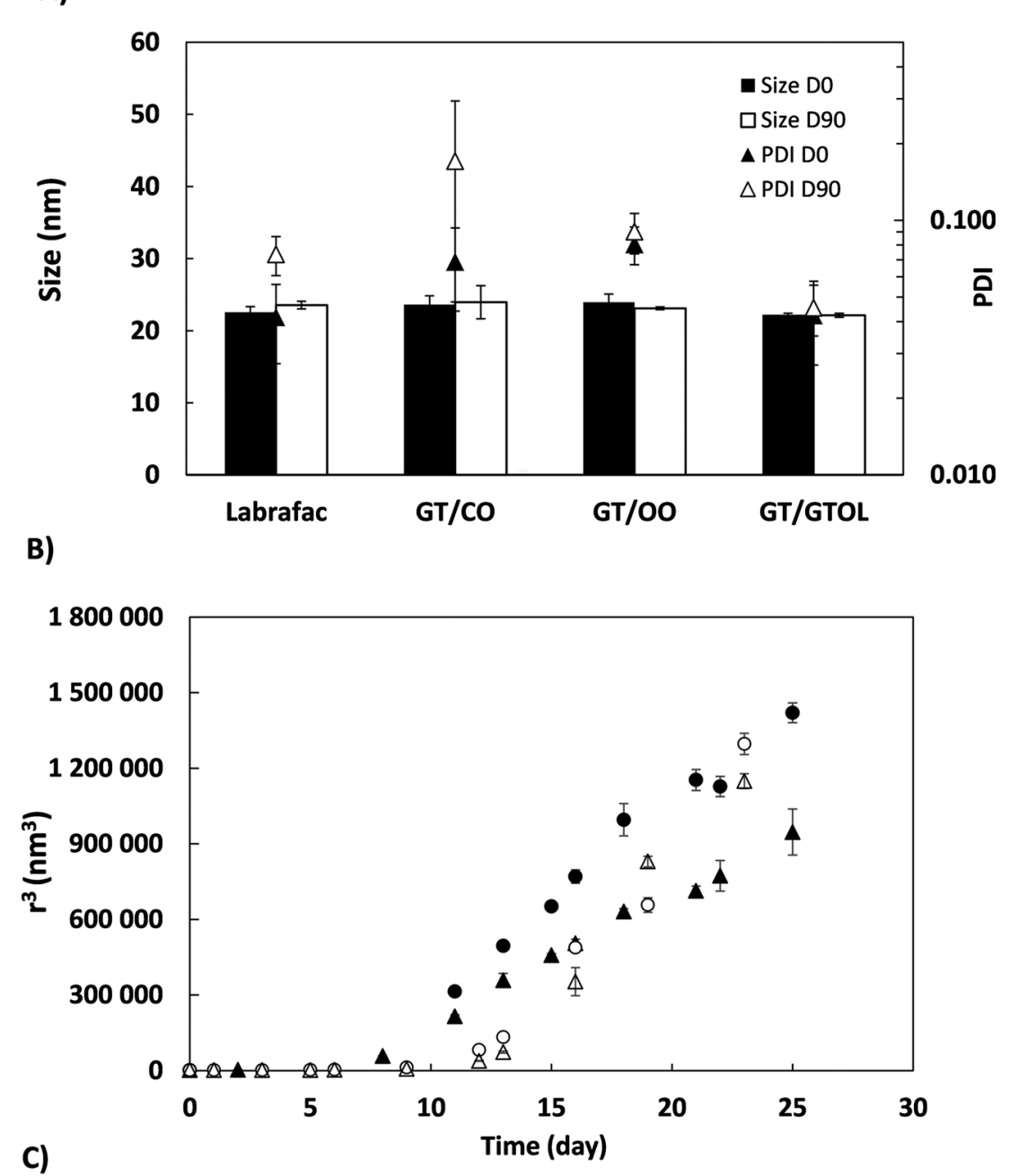

C)

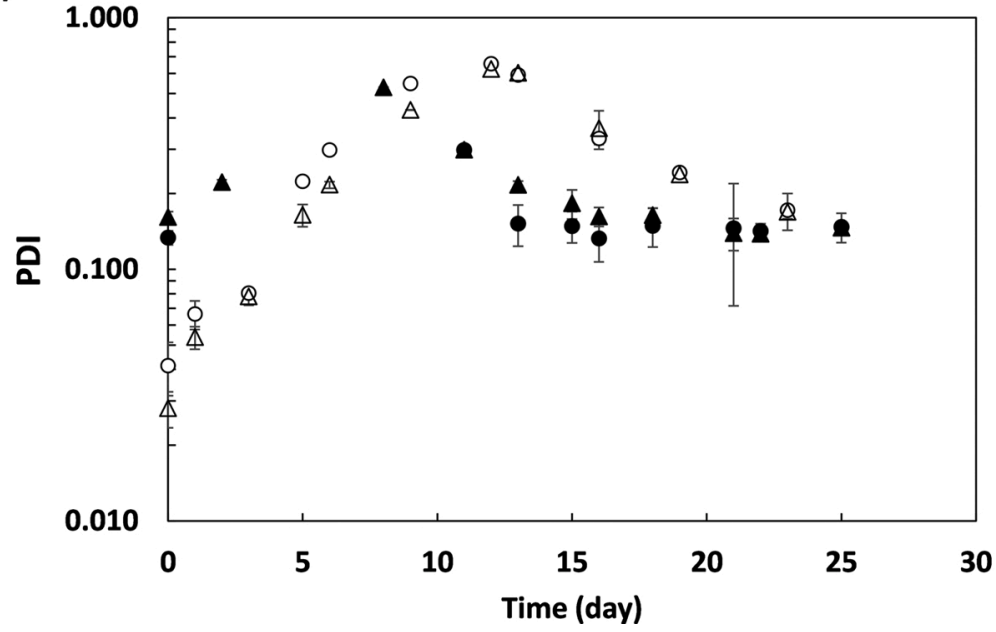

Fig. 7. Stability of nanoemulsions prepared at $37^{\circ} \mathrm{C}$ with a SOR and a WOR of 3.5 and 22, respectively. A: Long-term stability of nanoemulsions composed of Labrafac and of 1:1 mixtures of glyceryl trioctanoate with corn oil (GT/CO), olive oil (GT/OO) and glyceryl trioctanoate (GT/GTOL). Size and PDI were measured immediately after preparation (D0) and after 90 days of storage at $4{ }^{\circ} \mathrm{C}$ (D90). Results are expressed as mean values $\pm \mathrm{SD}$. Measurements were performed in triplicate

100 from at least two independent experiments. B: Cube of hydrodynamic radius of four batches of glyceryl trioctanoate

$\overline{0}$ nanoemulsion as a function of time. C: PDI of four batches of glyceryl trioctanoate nanoemulsion as a function of time. Results are expressed as mean values \pm SD. Measurements were performed in triplicate. stable over time [21]. This was explained by the entropy of mixing that dominates the Laplace pressure. This result demonstrates also that lipids droplets were composed of both MCT and LCT, since otherwise a destabilization would have been observed for droplet population composed of glyceryl trioctanoate. Thus, the LCT addition to glyceryl trioctanoate represents an interesting strategy to improve the physical stability of nanodroplets by limiting the Ostwald ripening rate.

\section{Conclusion}

This study emphasizes the major role of the oil type in the spontaneous nanoemulsification process. The molar volume of triglycerides represents a predictive factor for both the final size, the polydispersity and the stability of the emulsions prepared at a low temperature. Vm is correlated to the water solubility and the diffusion coefficient of oils molecules. Glyceryl trioctanoate characterized by a low Vm value facilitated both the spontaneous emulsification and the Ostwald 
ripening. Nanoemulsification at $37^{\circ} \mathrm{C}$ failed with vegetable oils due to long hydrocarbon chains (C18) which limits their diffusion in the continuous aqueous phase. Heating above the PIT was required to obtain LCT nanodroplets. Both stable and monodisperse nanoemulsions were obtained with Labrafac characterized by a Vm value of $535 \mathrm{~cm}^{3} / \mathrm{mol}$. Mixture of LCT with glyceryl trioctanoate allowed designing stable, fine and monodisperse nanoemulsions. This represents a promising approach to enlarge the variety of triglycerides in spontaneous emulsification processes at low temperature and without organic water-soluble solvent. This investigation paves the way to new strategies for the delivery of thermosensitive drugs and lipids such as polyunsaturated LCT.

\section{CRediT authorship contribution statement}

Yasmine Jamoussi: Investigation, Formal analysis, Writing - original draft. Taghrid Zaiter: Investigation. Catherine Desrumaux: Funding acquisition, Writing - review \& editing. Niyazi Acar: Funding acquisition, Writing - review \& editing. Yann Pellequer: Resources, Writing - review \& editing. Arnaud Béduneau: Supervision, Conceptualization, Methodology, Writing - original draft.

\section{Declaration of Competing Interest}

The authors report no declarations of interest.

\section{Acknowledgments}

This work was supported by grants from the Agence Nationale de la Recherche [ANR-11-LABEX-0021], the Conseil Régional Bourgogne, Franche-Comte (PARI grant), the FEDER (European Funding for Regional Economical Development) and the Fondation de France/Fondation de l'œil.

\section{References}

[1] C. Solans, P. Izquierdo, J. Nolla, N. Azemar, M.J. Garcia-Celma, Nano-emulsions, Curr. Opin. Colloid Interface Sci. 10 (3) (2005) 102-110, https://doi.org/10.1016/ j.cocis.2005.06.004.

[2] K. Shinoda, H. Saito, The effect of temperature on the phase equilibria and the types of dispersions of the ternary system composed of water, cyclohexane, and nonionic surfactant, J. Colloid Interface Sci. 26 (1) (1968) 70-74, https://doi.org/ 10.1016/0021-9797(68)90273-7.

[3] K. Bouchemal, S. Briançon, E. Perrier, H. Fessi, Nano-emulsion formulation using spontaneous emulsification: solvent, oil and surfactant optimisation, Int. J. Pharm. 280 (1-2) (2004) 241-251, https://doi.org/10.1016/j.ijpharm.2004.05.016.

[4] Y. Singh, et al., Nanoemulsion: concepts, development and applications in drug delivery, J. Control. Release Off. J. Control. Release Soc. 252 (2017) 28-49, https://doi.org/10.1016/j.jconrel.2017.03.008.

[5] G. Lefebvre, et al., Spontaneous nano-emulsification: process optimization and modeling for the prediction of the nanoemulsion's size and polydispersity, Int. J. Pharm. 534 (1-2) (2017) 220-228, https://doi.org/10.1016/j. ijpharm.2017.10.017.

[6] N. Anton, P. Saulnier, Adhesive water-in-oil nano-emulsions generated by the phase inversion temperature method, Soft Matter 9 (28) (2013) 6465-6474, https://doi.org/10.1039/C3SM51064F.

[7] C.W. Pouton, C.J.H. Porter, Formulation of lipid-based delivery systems for oral administration: materials, methods and strategies, Adv. Drug Deliv. Rev. 60 (6) (2008) 625-637, https://doi.org/10.1016/j.addr.2007.10.010.

[8] S. Peltier, J.-M. Oger, F. Lagarce, W. Couet, J.-P. Benoît, Enhanced oral paclitaxel bioavailability after administration of paclitaxel-loaded lipid nanocapsules, Pharm. Res. 23 (6) (2006) 1243-1250, https://doi.org/10.1007/s11095-006-0022-2.

[9] E. Roger, F. Lagarce, E. Garcion, J.-P. Benoit, Lipid nanocarriers improve paclitaxe transport throughout human intestinal epithelial cells by using vesicle-mediated transcytosis, J. Control. Release Off. J. Control. Release Soc. 140 (2) (2009) 174-181, https://doi.org/10.1016/j.jconrel.2009.08.010.
[10] R. Nieto Montesinos, A. Béduneau, Y. Pellequer, A. Lamprecht, Delivery of Pglycoprotein substrates using chemosensitizers and nanotechnology for selective and efficient therapeutic outcomes, J. Control. Release Off. J. Control. Release Soc. 161 (1) (2012) 50-61, https://doi.org/10.1016/j.jconrel.2012.04.034.

[11] D.H. Lohith Kumar, P. Sarkar, Encapsulation of bioactive compounds using nanoemulsions, Environ. Chem. Lett. 16 (1) (2018) 59-70, https://doi.org/ 10.1007/s10311-017-0663-x.

[12] S. Klek, et al., Home parenteral nutrition with an omega-3-fatty-acid-enriched MCT/LCT lipid emulsion in patients with chronic intestinal failure (the HOME study): study protocol for a randomized, controlled, multicenter, international clinical trial, Trials 20 (2019), https://doi.org/10.1186/s13063-019-3994-z.

[13] N. Anton, P. Gayet, J.-P. Benoit, P. Saulnier, Nano-emulsions and nanocapsules by the PIT method: an investigation on the role of the temperature cycling on the emulsion phase inversion, Int. J. Pharm. 344 (1) (2007) 44-52, https://doi.org/ 10.1016/j.ijpharm.2007.04.027.

[14] D.J. McClements, J. Rao, Food-grade nanoemulsions: formulation, fabrication, properties, performance, biological fate, and potential toxicity, Crit. Rev. Food Sci. Nutr. 51 (4) (2011) 285-330, https://doi.org/10.1080/10408398.2011.559558.

[15] J. Baloch, et al., Self-nanoemulsifying drug delivery system (SNEDDS) for improved oral bioavailability of chlorpromazine: in vitro and in vivo evaluation, Medicina (Mex.) 55 (5) (2019), https://doi.org/10.3390/medicina55050210.

[16] K. Hörmann, A. Zimmer, Drug delivery and drug targeting with parenteral lipid nanoemulsions - A review, J. Control. Release Off. J. Control. Release Soc. 223 (2016) 85-98, https://doi.org/10.1016/j.jconrel.2015.12.016.

[17] A.S. Kabalnov, K.N. Makarov, A.V. Pertzov, E.D. Shchukin, Ostwald ripening in emulsions: 2. Ostwald ripening in hydrocarbon emulsions: experimental verification of equation for absolute rates, J. Colloid Interface Sci. 138 (1) (1990) 98-104, https://doi.org/10.1016/0021-9797(90)90184-P.

[18] S. Ariyaprakai, S.R. Dungan, Influence of surfactant structure on the contribution of micelles to Ostwald ripening in oil-in-water emulsions, J. Colloid Interface Sci. 343 (1) (2010) 102-108, https://doi.org/10.1016/j.jcis.2009.11.034.

[19] J. Weiss, C. Canceliere, D.J. McClements, Mass transport phenomena in oil-inWater emulsions containing surfactant micelles: ostwald ripening, Langmuir 16 (17) (2000) 6833-6838, https://doi.org/10.1021/la991477v.

[20] Interfacial Tensions of Commercial Vegetable Oils with water - FISHER - 1985 -, Journal of Food Science - Wiley Online Library (2020), https://doi.org/10.1111/ j.1365-2621.1985.tb13052.x.

[21] T.J. Wooster, M. Golding, P. Sanguansri, Impact of oil type on nanoemulsion formation and Ostwald ripening stability, Langmuir ACS J. Surf. Colloids 24 (22) (2008) 12758-12765, https://doi.org/10.1021/la801685v.

[22] J.-L. Salager, N. Marquez, A. Graciaa, J. Lachaise, Partitioning of ethoxylated octylphenol surfactants in microemulsion-Oil-Water systems: influence of temperature and relation between partitioning coefficient and physicochemical formulation, Langmuir 16 (13) (2000) 5534-5539, https://doi.org/10.1021/ la9905517.

[23] A.M. Al-Sabagh, N.M. Nasser, M.A. Migahed, N.G. Kandil, Effect of chemical structure on the cloud point of some new non-ionic surfactants based on bisphenol in relation to their surface active properties, Egypt. J. Pet. 20 (2) (2011) 59-66, https://doi.org/10.1016/j.ejpe.2011.06.006.

[24] N. Anton, T.F. Vandamme, The universality of low-energy nano-emulsification, Int. J. Pharm. 377 (1-2) (2009) 142-147, https://doi.org/10.1016/j. ijpharm.2009.05.014.

[25] K. Shinoda, H. Arai, The correlation between phase inversion temperature in emulsion and cloud point in solution of nonionic emulsifier, J. Phys. Chem. 68 (12) (1964) 3485-3490, https://doi.org/10.1021/j100794a007.

[26] D. Morales, J.M. Gutiérrez, M.J. García-Celma, Y.C. Solans, A study of the relation between bicontinuous microemulsions and Oil/Water nano-emulsion formation, Langmuir 19 (18) (2003) 7196-7200, https://doi.org/10.1021/la0300737.

[27] D. Morales, C. Solans, J.M. Gutiérrez, M.J. Garcia-Celma, U. Olsson, Oil/Water droplet formation by temperature change in the Water/C16E6/Mineral oil system, Langmuir 22 (7) (2006) 3014-3020, https://doi.org/10.1021/la052324c.

[28] C. Solans, D. Morales, M. Homs, Spontaneous emulsification, Curr. Opin. Colloid Interface Sci. 22 (2016) 88-93, https://doi.org/10.1016/j.cocis.2016.03.002.

[29] B. Marten, M. Pfeuffer, J. Schrezenmeir, Medium-chain triglycerides, Int. Dairy J. 16 (11) (2006) 1374-1382, https://doi.org/10.1016/j.idairyj.2006.06.015.

[30] P. Taylor, Ostwald ripening in emulsions: estimation of solution thermodynamics of the disperse phase, Adv. Colloid Interface Sci. 106 (1) (2003) 261-285, https:// doi.org/10.1016/S0001-8686(03)00113-1.

[31] D. Boskou, Olive Oil: Chemistry and Technology, Elsevier, 2015.

[32] I.M. Lifshitz, V.V. Slyozov, The kinetics of precipitation from supersaturated solid solutions, J. Phys. Chem. Solids 19 (1) (1961) 35-50, https://doi.org/10.1016/ 0022-3697(61)90054-3.

[33] W.I. Higuchi, J. Misra, Physical degradation of emulsions via the molecular diffusion route and the possible prevention thereof, J. Pharm. Sci. 51 (5) (1962) 459-466, https://doi.org/10.1002/jps.2600510514. 